\title{
Numerical investigation of solid-liquid slurry flow through an upward-facing step
}

\author{
Gianandrea Vittorio Messa ${ }^{*}$, Stefano Malavasi \\ Dipt. Ingegneria Civile e Ambientale, Politecnico di Milano, Piazza Leonardo Da Vinci, 32, 20133 Milano, Italy. \\ ${ }^{*}$ Corresponding author. E-mail: gianandrea.messa@mail.polimi.it
}

\begin{abstract}
The flow of a solid-water mixture through an upward-facing step in a channel is numerically investigated. The effect of expansion ratio, mean solids volume fraction and particle diameter on the velocity field, pressure distribution and solid volume fraction field is studied. Expansion ratios of 0.50 and 0.67 , particle diameter of $125 \mu \mathrm{m}$ and $440 \mu \mathrm{m}$ and mean solid volume fraction between 0.05 and 0.20 are considered. Particle density is $2465 \mathrm{~kg} \mathrm{~m}^{-3}$. An Eulerian twofluid model is used to simulate the flow. Due to the lack of experimental data, the model was validated by comparison to other numerical investigations and to experimental data about the horizontal pipe case. Afterwards, it is studied the effect of the above mentioned parameters upon the degree of coupling between the phases and the extension of the disturbance region in the pressure and solid volume fraction fields downstream the step. Parameters of engineering interest, such as the reattachment length and the pressure recovery downstream the enlargement, are investigated.
\end{abstract}

Keywords: Two-fluid model; Numerical analysis; Slurries; Two-phase flow; Upward-facing step.

\section{INTRODUCTION}

Solid-liquid slurry flows are commonly encountered in many applications, in both civil and industrial engineering. The knowledge of their behavior is fundamental to ensure a correct and efficient functioning of the plants. In fact, the presence of solid particles in a liquid flow may affect the dissipation characteristics, procure damage to control and security devices, or cause the erosion of the ducts. Actually, the way in which the particles affect the dissipations is a very complex matter; typically, the presence of a solid phase produces an increase of the losses with respect to the single-phase flow case (Gillies et al., 2004; Kaushal et al., 2005; Shaan et al., 2000), but, under specific flow conditions (depending mainly on particle size and mean solids volume fraction), either negligible variations or even a decrease were observed (Shook and Bartosik, 1994; Shook and Roco, 1991).

The flow of solid-liquid mixtures is very complex. According to Doron and Barnea (1996), when a slurry flows in a horizontal pipe three major flow patterns can be identified as the flow rate decreases: a) fully suspended flow, in turn divided in pseudo-homogeneous flow and heterogeneous suspension flow; b) flow with a moving bed; c) flow with a stationary bed. In fully-suspended flow all the solid particles are suspended; if the velocity is high enough, the particles are uniformly distributed across the pipe cross-section (pseudo-homogeneous flow), otherwise there's a concentration gradient in the vertical direction (heterogeneous suspension flow). As the velocity decreases, the solid particles accumulate at the pipe bottom and form a packed bed moving along the pipe. The concentration of this layer is the maximum packing one (Doron and Barnea, 1996; Doron et al., 1987). At last, when the mixture velocity is insufficient to keep all the immersed particles in motion, a stationary deposit is observed at the bottom of the pipe.

Despite of its importance, the knowledge of this topic is far from been exhaustive. Most of the researches concern the case of flow through straight pipes or rectangular ducts and are experimental (Doron and Barnea, 1996; Doron et al., 1987; Gillies et al., 2004; Kaushal and Tomita, 2003; Kaushal et al., 2005; Matousek, 2000; Shaan et al., 2000; Shook and Roco,
1991; Vlasak and Chara, 2011; Vlasak et al., 2012). However, the technical and economic burden of lab test has led some authors to study the problem by means of computational fluid dynamics (Chen et al., 2009; Lahiri and Ghanta, 2010; Lin and Ebadian, 2008; Ling et al., 2003; Xiaowei and Liejin, 2010).

The literature currently available about the case of pipeline fittings - such as bends, sudden enlargements, sudden contractions, perforated plates and valves - is quite poor, although they are integral parts of the plants. In these cases, the greater complexity of the phenomenon makes it very difficult to perform experimental investigations (Founti and Klipfel, 1998) and the use of numerical techniques is almost the only possible way to study the flow field in detail. Founti and Klipfel (1998) analyzed the flow of a dilute mixture of glass particles and diesel oil through a sudden enlargement in a pipe both experimentally and numerically, using an Eulerian-Lagrangian model. They focused on the mean and fluctuating velocity distributions of the two phases and considered slurries with particle volumetric concentration below 0.05. Badr et al. $(2005,2008)$ and Habib et al. $(2004,2007)$ studied the flow of sand particles and water through sudden contractions in vertical pipes. They made use of an Eulerian-Lagrangian model under the hypothesis of one-way coupling between the phases, with the aim to predict the erosion of the system. Mohanarangam and Tu (2009) performed simulations about the flow of a mixture of glass particles and diesel oil through a backward-facing step using an Eulerian two-fluid model, and analyzed the dependence of the mean and fluctuating velocities of the solid phase upon the particle Stokes number, i.e. the ratio between the particle relaxation time and a time characteristic of the fluid motion. Frawley et al. (2010) compared Eulerian-Eulerian and Eulerian-Lagrangian models in the simulation of the flow of the same mixture of glass particles and diesel oil through a sudden enlargement in a circular pipe. They focused their attention on the velocity field and found that the discrepancy in the predictions of the mean velocity profiles of both phases obtained with the two models is insignificant and that the Eulerian-Eulerian model yields more accurate results in term of turbulent kinetic energy. Pathak (2011) made use of an algebraic slip model to study a solidwater slurry flow around a rectangular block at the bottom of a 
channel, and analyzed on the effect of particle diameter upon the mixture velocity, the volume fraction of the solid phase and the turbulence intensity.

In the present work a solid-liquid slurry flow through an upward-facing step in a channel is studied (Fig. 1). The height of the upstream channel is $26 \mathrm{~mm}$, that of the downstream one is set either to $39 \mathrm{~mm}$ or to $52 \mathrm{~mm}$ (corresponding to expansion ratios $\beta$ equal to 0.67 and 0.50 respectively). The carrier fluid is water, and two kinds of particles (density $\rho_{p}=2465 \mathrm{~kg} \mathrm{~m}^{-3}$; diameter $d_{p}$ equal to 125 and $440 \mu \mathrm{m}$ ) have been considered. The mean volumetric concentration of solid particles $\overline{\alpha_{p}}$ was varied between 0.05 and 0.20 . In all cases, the slurry bulk-mean inlet velocity (i.e. the velocity in the small duct), referred to as $\bar{U}_{m}$, is $4 \mathrm{~m} / \mathrm{s}$. The effects of mean solids volume fraction, particle diameter and expansion ratio of the enlargement upon the velocity field, the pressure profile and the local solids volume fraction distribution are investigated.

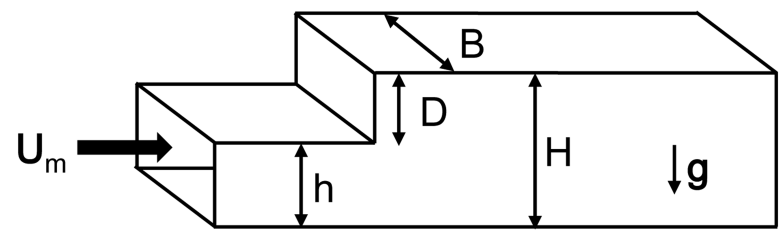

Fig. 1. Geometrical configuration of the problem.

\section{MATHEMATICAL MODEL AND VALIDATION Two-fluid model}

In the present work we made use of an Eulerian two-fluid model implemented in the commercial code PHOENICS 2009 by CHAM Ltd. This model, which is described in Spalding (1980), is based on the concept of interpenetrating continua, and solves the mass and momentum conservation equations for the two phases. The flow is assumed to be steady, thus the phase continuity equation for phase $j=C, p$ has the following form:

$\nabla \cdot\left(\alpha_{j} \rho_{j} \vec{U}_{j}-\Gamma_{\alpha j} \nabla \alpha_{j}\right)=0$

where $\alpha_{j}$ is the volume fraction, $\rho_{j}$ is the density, $\vec{U}_{j}$ is the velocity, and $\Gamma_{\alpha j}$ is a phase diffusion coefficient, which appears in the phase diffusion term that represents the turbulent flux associated with correlations between fluctuating velocity and volume fraction. The phase diffusion fluxes are modeled in terms of a gradient diffusion approximation with the phasediffusion coefficient $\Gamma_{\alpha j}$ given by:

$\Gamma_{\alpha j}=\frac{\rho_{j} v_{T}}{\sigma_{\alpha p}^{T}}$

where $v_{T}$ is the turbulent kinematic viscosity of the carrier fluid phase, and $\sigma_{\alpha p}^{T}$ is the turbulent Schmidt number for volume fractions. The mean global continuity is given by the equation that states that the two volume fractions must sum to unity.

The momentum equation for $u_{j, i}$, the $i$-velocity component of phase $j=C, p$, is:

$$
\begin{aligned}
& \nabla \cdot\left(\alpha_{j} \rho_{j} \vec{U}_{j} u_{j, i}-u_{j, i} \Gamma_{\alpha j} \nabla \alpha_{j}-\alpha_{j} \Gamma_{u j, i} \nabla u_{i, j}\right)= \\
& =-\alpha_{j} \frac{\partial P}{\partial x_{i}}+\alpha_{j} \rho_{j} \mathrm{~g}_{i}+M_{j, i},
\end{aligned}
$$

where $P$ is the pressure, shared by the phases, $\mathrm{g}_{i}$ is the $i$ component of the gravitational acceleration, and $M_{j, i}$ is a term accounting for the interfacial momentum transfer within the phases, which will be discussed later. Two diffusion terms

appear on the left side of Eq. (3): the former $\nabla \cdot\left(u_{j, i} \Gamma_{\alpha j} \nabla \alpha_{j}\right)$ accounts for the transport of momentum brought about by the turbulent dispersion of the phase; whereas the latter $\nabla \cdot\left(\alpha_{j} \Gamma_{u j} \nabla u_{j, i}\right)$ is due to within-phase molecular and turbulent diffusion. As in previous works dealing with similar kind of flows (Marjanovic et al., 1999), the diffusion coefficient $\Gamma_{u j}$ is modeled as:

$\Gamma_{u j}=\rho_{j}\left(v+v_{T}\right)$,

where $v$ is the kinematic viscosity coefficient of the carrier fluid phase.

The term $M_{j, i}$ in the momentum equation (Eq.(3)) is the $i$ component of the force per unit volume acting on a suspension of particles, which is often referred to as "generalized drag" (Enwald et al., 1996; Ishii and Mishima, 1984). The generalized drag is normally divided as: stationary drag, Saffman and Magnus lift, added mass, and Basset (or "history") force. The two-fluid model represents the turbulent dispersion of particles by means of a turbulent diffusion term in the phasic continuity equation (Eq. (1)), and so an explicit turbulent-dispersion force term makes no appearance in the momentum equation. In the present work only the stationary drag was included, since all other contributions are negligible for the kind of flow considered and were found not to contribute significantly to particle motion (as already reported by Habib et al., 2004). Therefore, the $i$-component of the generalized drag is given by:

$M_{j, i}=\frac{3}{4 d_{p}} \alpha_{p} \rho_{C} C_{d}\left|u_{s, i}\right| u_{s, i}$,

where $d_{p}$ is the particle diameter, $C_{d}$ is the drag coefficient and $u_{s, i}$ is the $i$-component of the slip velocity between the phases, equal to $u_{p, i}-u_{C, i}$ for $j=C$ and to $u_{C, i}-u_{p, i}$ for $j=p$. The drag coefficient $C_{d}$ is related to the particle Reynolds number $R_{p}=d_{p}\left|\vec{U}_{p}-\vec{U}_{C}\right| / v$ by the following correlation attributed to Clift and Gauvin and reported in Clift et al. (1978):

$C_{d}=\frac{24}{R_{p}}\left(1+0.15 R_{p}^{0.687}\right)+\frac{0.42}{1+4.25 \cdot 10^{4} R_{p}^{-1.16}}$.

which is claimed to be valid for $R_{p}<310^{5}$.

Turbulence is assumed to be a property of the carrier fluid phase, and turbulent flow is modeled using the k- $\varepsilon$ RNG model, which, taking care of the effect of rapid strain in complex flow, it capable of predicting the gross flow behaviour in recirculating region (Pathak, 2011). The same choice was made by other authors who investigated similar kind of flows (Erdal and Anderssont, 1997; Frawley et al., 2010; Pathak, 2011). 

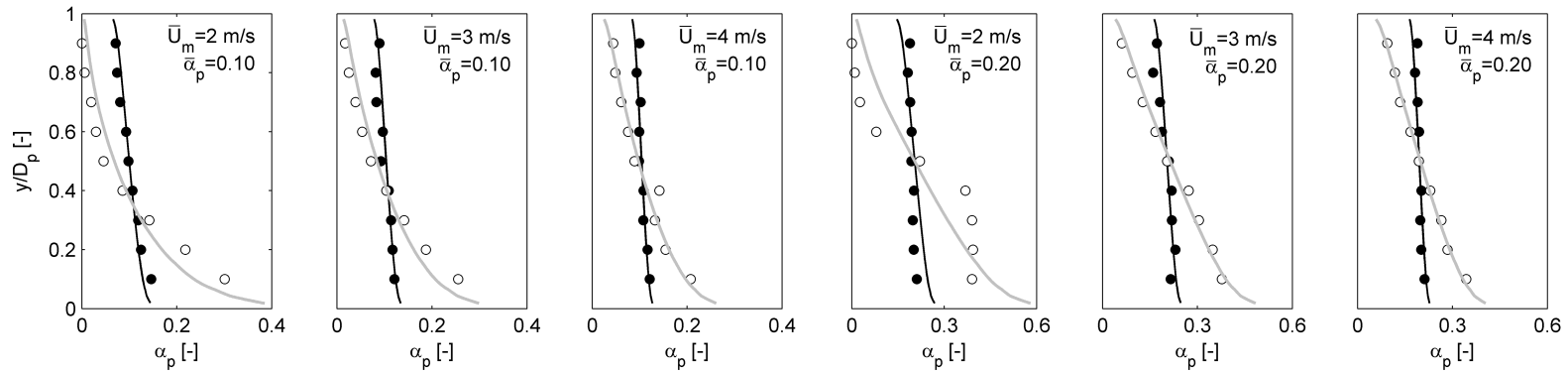

Fig. 2. Solid volume fraction along the vertical diameter in a horizontal pipe: comparison between our numerical predictions and the experimental data of Kaushal et al., 2005 (०: experimental, $d_{p}=125 \mu \mathrm{m}$; O: experimental, $d_{p}=440 \mu \mathrm{m} ;-$ numerical, $d_{p}=125 \mu \mathrm{m} ; \square$ numerical, $\left.d_{p}=440 \mu \mathrm{m}\right)$.

\section{Calibration of the model and validation}

The solutions produced by the two-fluid model depend upon the value assigned to the turbulent Schmidt number for volume fractions $\sigma_{\alpha p}^{T}$. Suitable values for such empirical constant were determined by comparison of model predictions with the experimental data of Kaushal et al. (2005) for solid-liquid slurry flow through a horizontal pipe. The turbulent Schmidt number for volume fraction $\sigma_{\alpha p}^{T}$ was found to have a strong effect on the volume fraction profile. Several simulations were performed, and a value of $\sigma_{\alpha p}^{T}$ equal to 0.25 was found to provide a good match between our numerical predictions and the experimental data for slurry bulk-mean inlet velocity $\bar{U}_{m}$ (i.e. the velocity in the pipe) between 2 and $4 \mathrm{~m} \mathrm{~s}^{-1}$, mean solids volume fraction $\bar{\alpha}_{p}$ equal to 0.10 and 0.20 , and particle size $d_{p}$ equal to $125 \mu \mathrm{m}$ and $440 \mu \mathrm{m}$ (Fig. 2). Particle density is $2465 \mathrm{~kg} / \mathrm{m}^{3}$. The experimental data reveal that, for $\bar{U}_{m}=2 \mathrm{~m} \mathrm{~s}^{-1}, \bar{\alpha}_{p}=0.20$ and $d_{p}=$ $440 \mu \mathrm{m}$, a bed of particles forms at the bottom of the pipe. The concentration of this layer approaches the maximum packing one. The two-fluid model is unable to reproduce this phenomenon, and this limitation is shared also by other Eulerian models implemented in commercial codes (Chen et al., 2009; Ling et al., 2003). Special attention was therefore paid to guarantee that, in our simulations concerning the upward-facing step case, the inlet velocity $\bar{U}_{m}$, the mean solids volume fraction $\bar{\alpha}_{p}$, and the particle characteristics (i.e. in terms of size $d_{p}$ and density $\rho_{p}$ ) were suitable to keep all the solid particles suspended.

\section{Computational domain and boundary conditions}

The step was modeled in two dimensions. Preliminary tests (Messa and Malavasi, 2012) showed that the results of a twodimensional test are a good approximation of a threedimensional test in the central part of the channel if the aspect ratio of the cross-section of the downstream channel $B / H$ is sufficiently large, say about $15-20$ or more. Similar observations are reported by Fessler and Eaton (1997). Fig. 3 shows the configuration of the computational domain. At the inlet, no slip is assumed between the phases; the same uniform axial velocity distribution equal to $\bar{U}_{m}$ is applied to the fluid and the particles, while the distributions of the turbulent kinetic energy and of its dissipation rate were derived from a turbulent intensity of $5 \%$ and an inlet mixing length equal to $7 \%$ of the hydraulic diameter. Also the inlet volume fraction of the solids is taken as uniformly distributed with value $\bar{\alpha}_{p}$. At the outlet, the normal gradients of all variables and the value of the pressure were set to zero.

The inlet boundary was located sufficiently far upstream the step $(150 \mathrm{~h})$ for full-developed flow conditions to have been achieved, which is typically $65 \mathrm{~h}$ downstream the inlet. This distance is the same order of magnitude of that of 50 pipe diameters indicated by other authors (Lin and Ebadian, 2008; Ling et al., 2003; Pathak, 2011; Xiaowei and Liejin, 2010) for the circular pipe case. $210 H$ was modeled downstream the step to provide an accurate estimation of the region in which the pressure and volume fraction distributions are affected by the step. Specific tests were performed to guarantee that such distance was sufficient whatever the flow conditions considered.

No-slip conditions were imposed on the walls. In the nearwall region, the non-equilibrium wall function of Launder and Spalding (1972) was set. Both Koronaki et al. (2001) and Siriboonluckul and Juntasaro (2007) report that, for separated flows, the k- $\varepsilon$ RNG turbulence model with non-equilibrium wall functions works better in the near-wall regions with respect to the standard wall functions. In all cases, it was checked that the application of such condition was consistent with the non-dimensional distance of the first grid points from the walls.

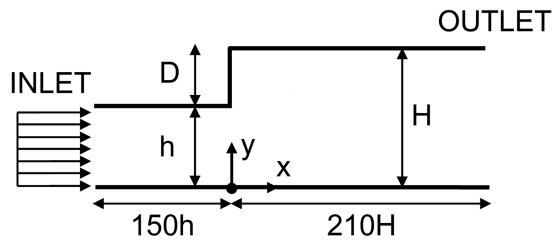

Fig. 3. Configuration of the computational domain.

\section{Computational methodology and consistency of the numerical solution}

PHOENICS uses the finite-volume method. The calculations were performed following the elliptic-staggered formulation in which the scalar variables are evaluated at the cell centers and the velocity components at the cell faces. Central differencing is employed for the diffusion terms, while the convection terms are discretized using the hybrid differencing scheme. The SIMPLEST algorithm is then used to solve the finite-volume equations.

A block-structured mesh was adopted to discretize the domain. Along the flow direction, the grid consisted of 1300 cells, concentrated near the step wall. Along the vertical direction, control volumes of about $0.75 \mathrm{~mm}$ size (i.e. 53 and 70 cells for the case of $\beta=0.67$ and 0.50 respectively) were used. A grid sensitivity study was made to choose the optimum discretiza- 
tion in the investigations. The tests were performed for the case of $\beta=0.50, \bar{\alpha}_{p}=0.10$ and $d_{p}=125 \mu \mathrm{m}$. Three different meshes, namely $620 \times 34$ (620 along the flow direction and 34 along the vertical one), $920 \times 50$ and $1330 \times 70$ were considered, and the grid convergence index of Roache (1998) was employed to quantify the uncertainty of grid convergence. Using this method, the numerical uncertainty in the $1330 \times 70$ grid solution for the reattachment length was estimated to be $0.8 \%$. Other tests confirm that further increase in mesh resolution has negligible effect on the reattachment length as well as the velocity and solid volume fraction profiles in different sections of the channel. The $1330 \times 70$ mesh was therefore used. As further proof of the consistency of the numerical model, we simulated single-phase flow through a sudden enlargement in a horizontal pipe. In particular, we reproduced the scenarios considered by Founti and Klipfel (1998) and Poole and Escudier (2004). The comparison of the distributions of axial velocity and turbulent kinetic energy revealed good agreement in both cases.

\section{RESULTS OF THE NUMERICAL PREDICTION Velocity distribution}

In order to investigate the velocity field of the slurry, reference is usually made to the mass-averaged mixture velocity, defined as:

$\vec{U}_{m}=\frac{\alpha_{p} \rho_{p} \vec{U}_{p}+\alpha_{C} \rho_{C} \vec{U}_{C}}{\alpha_{p} \rho_{p}+\alpha_{C} \rho_{C}}$.

As in the single phase case, the flow separates at the corner of the expansion and reattaches to the upper wall further downstream; recirculation takes place downstream the step lip (Fig. 4 ). The vertical profiles of the axial mixture velocity at different locations along the streamwise direction are provided as supplementary material to the printed paper (Figs. S1 to S3).

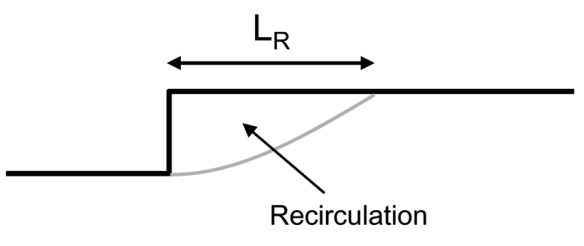

Fig. 4. Qualitative sketch of the flow field: recirculation region and reattachment length.

The slip velocity $\vec{U}_{s}=\vec{U}_{C}-\vec{U}_{p}$, which can be taken as an indicator of the degree of coupling between the phases, is investigated as a function of different parameters. The component of the slip velocity along the axial direction is averaged over the channel cross-sectional area and normalized by the slurry inlet velocity $\bar{U}_{m}$; the results are presented in Fig. 5 for a length of $50 \mathrm{D}$ downstream the step. The non negligible values of $\bar{u}_{s, x} / \bar{U}_{m}$ indicate that the degree of coupling between the phases can be significant, and therefore a one-way coupling model may not be suitable to correctly reproduce the phenomenon. The negative values of $\bar{u}_{s, x} / \bar{U}_{m}$ downstream the step indicate that the particles tend to move faster than the carrier fluid; this may happen because, due to their higher inertia, the particles not respond readily unlike the carrier phase to adverse pressure gradients found in that region. The comparison between the two particle diameters seems to confirm such interpretation (Fig. 5c). The slip between the phases appears to decrease as the mean particle volume fraction increases whatever the expansion ratio considered and to increase with the expansion ratio (Fig. 5a, b). These features appear to be in agreement with the numerical simulations of Mohanarangam and $\mathrm{Tu}$ (2009) and Marjanovic et al. (1999) and the experimental data of Founti and Klipfel (1998).

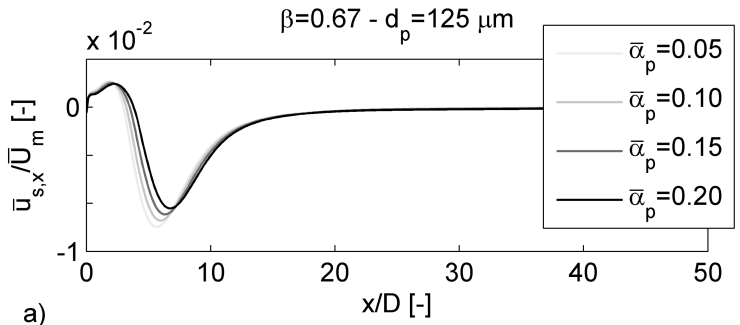

a)
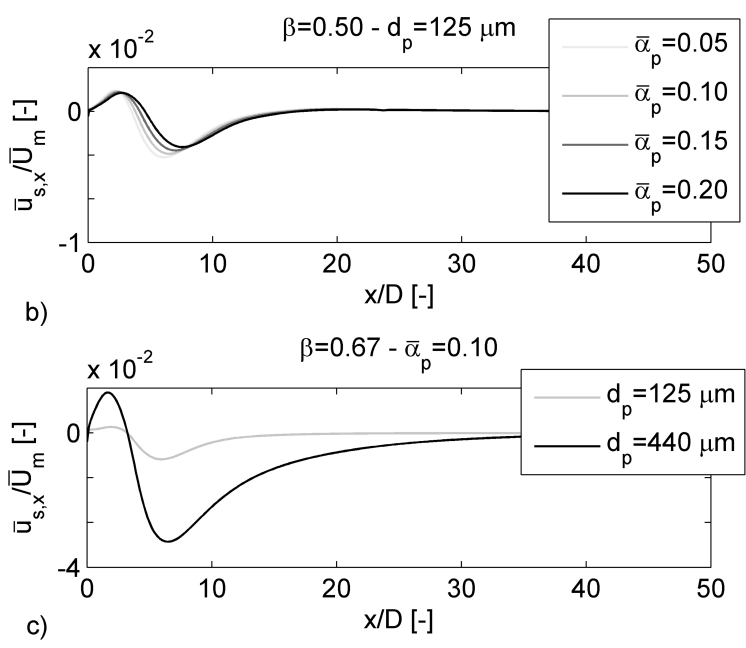

Fig. 5. Ratio between the slip velocity averaged over the channel cross-sectional area and the bulk-mean mixture velocity downstream the step for different values of expansion ratio, mean solid volume fraction, and particle diameter.

The dependence of the reattachment length upon the expansion ratio, particle diameter and mean particle volume fraction is studied. The results are reported in Fig. 6, where the reattachment length $L_{R}$ is normalized by the step height $D . L_{R} / D$ increases as $\beta$ decreases whatever the composition of the slurry. Moreover, $L_{R} / D$ increases with $\bar{\alpha}_{p}$ within the range considered, assuming higher values with respect to the single phase case (the black filled points in Fig. 6) $\beta$ being the same. The effect of $\bar{\alpha}_{p}$ seems to be stronger for the smaller particles. Our results appear compatible to those of Founti and Klipfel (1998), who studied the flow of diesel oil and glass particles with low volumetric loadings through a sudden enlargement in a circular pipe. However, a direct comparison cannot be strictly made since the two investigations refer to different operative conditions.

\section{Pressure profile}

A typical pressure profile for the mixture close to the step is shown in Fig. 7. The losses due to friction in the smaller channel cause the pressure to decline. As the step is reached, the 
mixture is decelerated in the larger channel and there occurs a sudden rise in pressure. Then, the pressure decreases again due to friction in the larger channel.

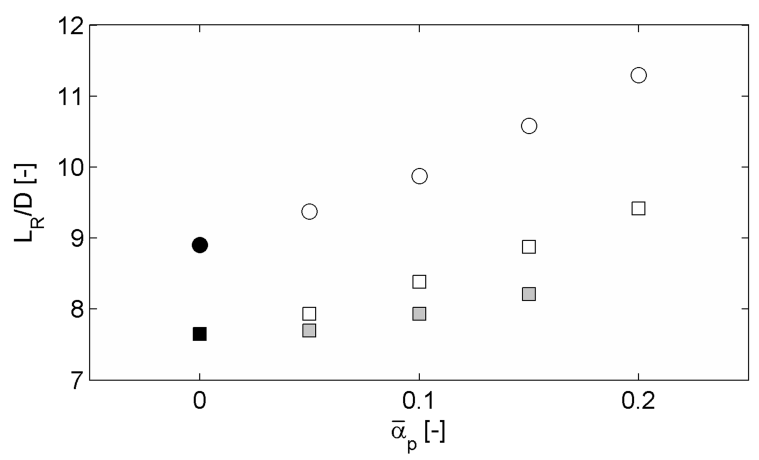

Fig. 6. Trend of the reattachment length as a function of the mean solid volume fraction for different values of expansion ratio and particle diameter $\left(\boldsymbol{O}: \beta=0.50\right.$, single phase; $\bigcirc: \beta=0.50, d_{p}=125$ $\mu \mathrm{m} ; \mathbf{\square}: \beta=0.67$, single phase; $\square: \beta=0.67, d_{p}=125 \mu \mathrm{m} ; \square: \beta=$ $\left.0.67, d_{p}=440 \mu \mathrm{m}\right)$.

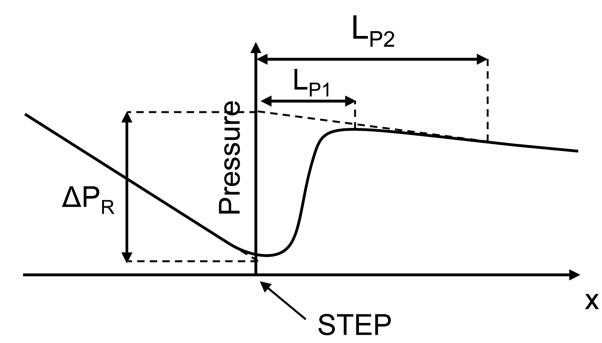

Fig. 7. Qualitative pressure profile and identification of the transition lengths.

The dependence of the pressure recovery $\Delta P_{R}$, defined as the difference in pressure when the fully developed pressure gradient lines are extrapolated to the step section (Fig. 7), upon the expansion ratio, particle diameter and mean particle volume fraction is considered. The results, displayed in Fig. 8, show that the pressure recovery increases with $\bar{\alpha}_{p}$ and it's higher than that of the single phase flow case. This may be due to the additional momentum transfer from solid particles which are flowing at higher velocity in the region downstream the step. The increase in pressure doesn't seem to depend significantly on the particle diameter.

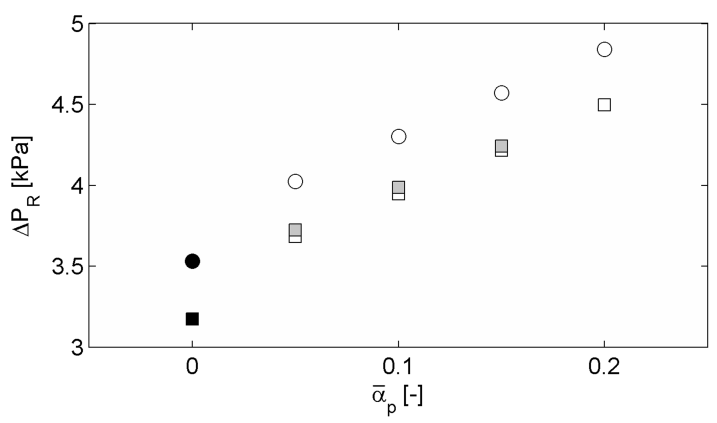

Fig. 8. Trend of the pressure recovery as a function of the mean solid volume fraction for different values of expansion ratio and particle diameter $\left(\bullet: \beta=0.50\right.$, single phase; $\bigcirc: \beta=0.50, d_{p}=125$ $\mu \mathrm{m} ; \mathbf{\square}: \beta=0.67$, single phase; $\square: \beta=0.67, d_{p}=125 \mu \mathrm{m} ; \square: \beta=$ $\left.0.67, d_{p}=440 \mu \mathrm{m}\right)$.
According to different authors (Duz, 2007; Marjanovic et al., 1999), two pressure transition lengths can be defined:

- $L_{P 1}$ : From the step to the point where the pressure reaches its maximum value. As shown in Fig. 9a, the non-dimensional length $L_{P 1} / D$, obtained dividing $L_{P 1}$ by the step height $D$, increases with the mean particle volume fraction $\bar{\alpha}_{p}$ and decreases with the expansion ratio $\beta$ and the particle diameter $d_{p}$.

- $L_{P 2}$ : From the step to the point where the pressure gradient reaches a constant value. This length is not easy to determine since a criterion to characterize the establishment of a constant pressure gradient - which is approached only asymptotically - must be set. In the present work we define $L_{P 2}$ as the distance from the step in which the local pressure gradient equals its asymptotic value unless than a given relative tolerance $\varepsilon_{r e l}$. Fig. $9 \mathrm{~b}$ shows the dimensionless length $L_{P 2} / D$ as a function of the mean solid volume fraction $\bar{\alpha}_{p}$ for different expansion ratios $\beta$ and particle diameter $d_{p}$ when $\varepsilon_{r e l}=0.01$; as expected, $L_{P 2} / D$ is larger than $L_{P 1} / D$, and increases with $\bar{\alpha}_{p}$ and $\beta$. Unlike $L_{P 1} / D, L_{P 2} / D$ increases with $d_{p}$ but for both parameters the effect of such variable seems to be of minor importance. The values reported in $L_{P 2} / D$ do not appear very sensitive to the tolerance $\varepsilon_{r e l}$; setting $\varepsilon_{r e l}=$ $=0.001$ produces a variation of $L_{P 2} / D$ lower than $2.5 \%$.
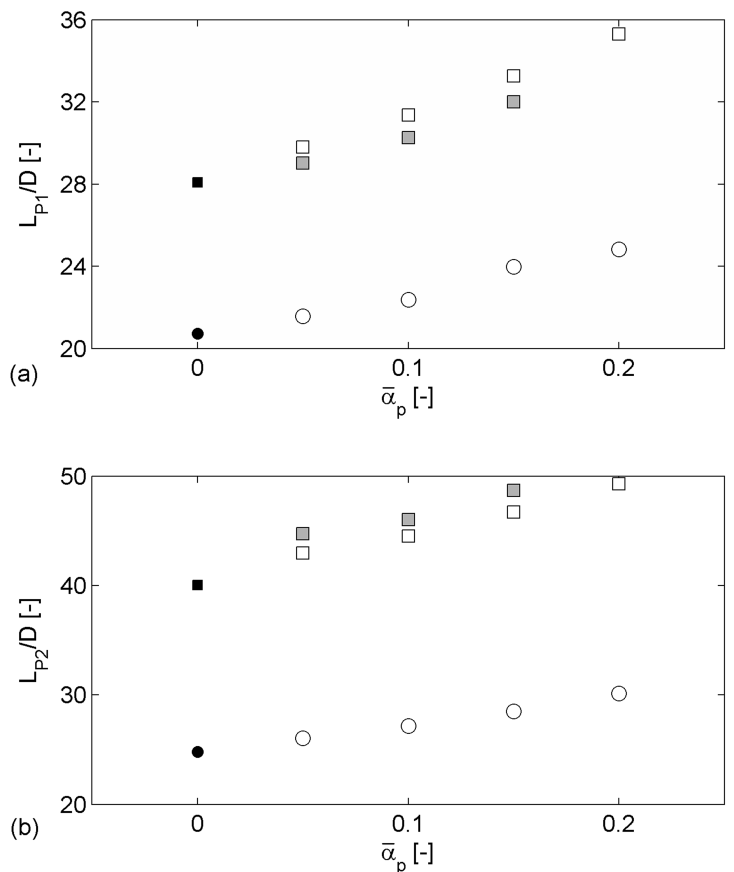

Fig. 9. Trend of the pressure transition lengths $L_{P 1} / D$ (a) and $L_{P 2} / D$ (b) as a function of the mean solid volume fraction for different values of expansion ratio and particle diameter $(\boldsymbol{O}: \beta=0.50$, single phase; $\bigcirc: \beta=0.50, d_{p}=125 \mu \mathrm{m} ; \mathbf{\square}: \beta=0.67$, single phase; $\square: \beta=$ $\left.0.67, d_{p}=125 \mu \mathrm{m} ; \square: \beta=0.67, d_{p}=440 \mu \mathrm{m}\right)$.

\section{Solids volume fraction distribution}

The distribution of the solid volume fraction upstream the step shows the typical trend of the heterogeneous suspension flow in a straight pipe (Doron and Barnea, 1996), and seems not to be affected by the step even at $x / D=-1$. For $x / D$ between 0 and about 10 , the flow recirculates. In the upper part of the 

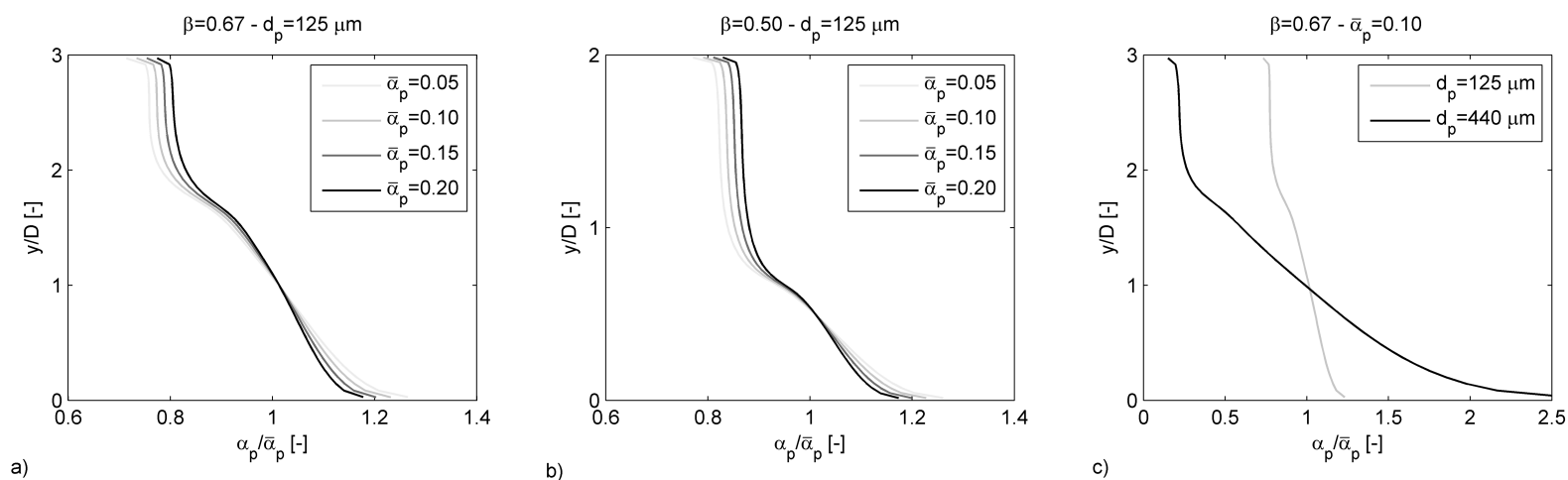

Fig. 10. Solid volume fraction profiles downstream the step $(x / D=5)$ as a function of expansion ratio, particle diameter and mean particle volume fraction.

channel, the gradient of the volume fraction is low because of wake vortices while, in the lower part, particles are driven upwards due to the high velocity gradients. Further downstream the enlargement the effect of the abrupt change in geometry disappears and the volume fraction profiles become similar to that of the smaller channel. Similar observations were made by Pathak (2011) studying the flow around an obstruction in a channel. The solids volume fraction profiles at different locations along the streamwise directions are provided as supplementary material to the printed paper (Figs. S4 to S6).

A parametric analysis of the solid volume fraction distribution downstream the step was performed. Fig. 10 reports the $\alpha_{p} / \bar{\alpha}_{p}$ profiles at $x / D=5$ for: a) $\beta=0.67, d_{p}=125 \mu \mathrm{m}$ and different values of $\bar{\alpha}_{p}$; b) $\beta=0.50, d_{p} 125 \mu \mathrm{m}$ and different values of $\bar{\alpha}_{p}$; c) $\beta=0.67, \bar{\alpha}_{p}=0.10$ and the two particle diameters. As shown in Fig. 10a, b the profile becomes flatter as the slurry concentration increases for both the height ratios. This may be due to the fact that, in this section, the degree of coupling between the phases increases with the mean solid volume fraction (since, as shown in Fig. 5a,b the axial slip velocity decreases with $\bar{\alpha}_{p}$ ), and therefore the percentage of particles dragged into the vortex increases. The solid volumetric fraction profile is very sensitive to the particle diameter (Fig. 10c): the heaviest particles are characterized by a higher Stokes number and therefore tend to occupy the lower part of the channel, being less affected by the expansion due to the enlargement.

As the particles approach the step, they are dragged upwards from the expanding jet and then fall down again until the balance between the effects of gravity and turbulence leads to fully-developed conditions. Consequently, the solid volume fraction at the bottom of the larger channel $\alpha_{B}$ decreases to a minimum then increases to a maximum (indeed the maximum of $\alpha_{p}$ in the whole domain) and finally reaches a constant value, as qualitatively depicted in Fig. 11a. The maximum value of $\alpha_{p}$ is a crucial parameter to ensure the reliability of the results. In fact, as already discussed, the need to avoid regions in which the particle accumulates for the two-phase model to be applicable implies that the solid volume fraction must be kept sufficiently below the maximum packing one everywhere; therefore, a check on the maximum value of $\alpha_{p}$ is a simple way to guarantee the consistency of the numerical solution.

The extension of the region in which the solid volume fraction is affected by the step is hard to quantify because a fullydeveloped concentration profile is reached only asymptotically. An estimation of such parameter - although by defect - can be taken as the distance $L_{\alpha}$ of the maximum of $\alpha_{p}$ at the bottom of the large channel from the step, much easier to determine. The dependence of the dimensionless parameter $L_{\alpha} / D$ upon the expansion ratio, particle diameter and mean particle volume fraction is considered. The results, reported in Fig. 11b, show that $L_{\alpha} / D$ is about 200 for $\beta=0.67$ and $d_{p}=125 \mu \mathrm{m}$; about 135 for $\beta=0.50$ and $d_{p}=125 \mu \mathrm{m}$; and about 165 for $\beta=0.67$ and $d_{p}$ $=440 \mu \mathrm{m}$; the influence of $\bar{\alpha}_{p}$ appears to be minor. The comparison between Fig. $12 \mathrm{~b}$ and $9 \mathrm{~b}$ seems therefore to indicate that the solid volume fraction distribution reaches fully developed conditions at a longer distance downstream the step with respect to the pressure field.

(a)
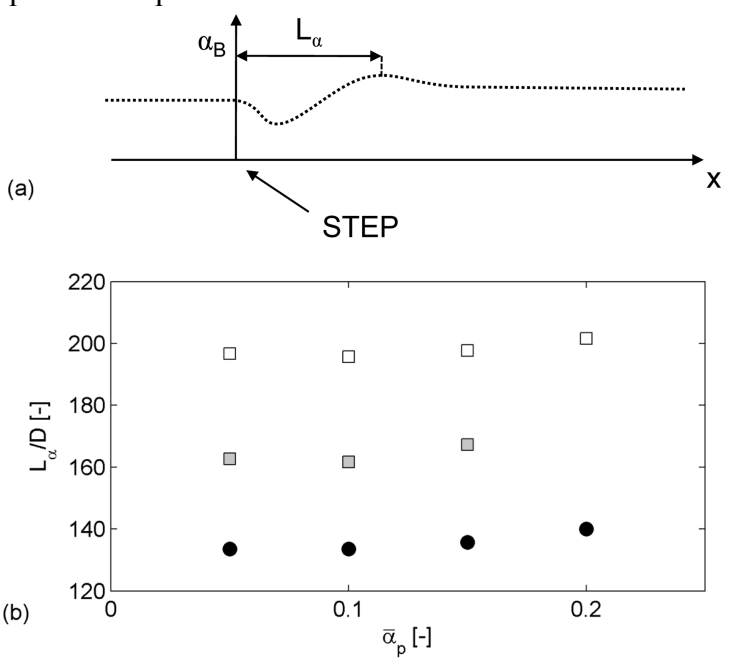

Fig. 11 (a) Qualitative trend of the solid volumetric fraction at the bottom of the channel $\alpha_{B}$ and identification of the dimensionless length $L_{\alpha}$; (b) Trend of the dimensionless length $L_{\alpha} / D$ as a function of the mean solid volume fraction for different values of expansion ratio and particle diameter.

\section{CONCLUSIONS}

The solid-water slurry flow through an upward-facing step has been studied numerically by means of an Eulerian two-fluid model. The effect of the expansion ratio, the particle diameter and the mean solid volume fraction has been investigated. The model has been validated through a comparison to experimental data about the flow through a horizontal pipe for different values of mixture velocity, mean solid volume fraction and particle diameter. As already reported in previous works, the two-fluid model was found to provide good prediction of the behavior of 
fully-suspended mixtures, but it fails when the solid particles accumulate and a packed bed is formed at the bottom of the channel (Fig. 2). Based on our investigations, the following conclusions can be drawn:

- The mixture separates at the corner of the expansion and reattaches to the upper wall further downstream; recirculation takes place downstream the step lip (Fig. 4). The degree of coupling between the phases increases with the mean solids volume fraction but decreases as the particle diameter increases, probably due to the higher inertia of the particles (Fig. 5). The reattachment length is higher to that of single-phase flow, and increases with the mean solids volume fraction, but decreases as the particle diameter and the expansion ratio increase (Fig. 6).

- Probably due to the additional momentum transfer from solid particles, the pressure recovery due to the expansion of the jet is higher than that of the single-phase case and increases with the solids volume fraction, but seems not to be affected by particle diameter (Fig. 8). Also the length of the disturbance region of the pressure field was found to increase with the solid volume fraction (Fig. 9).

- Downstream the step, the particles are dragged upwards from the expanding jet and then fall down again until a fully developed state is reached again. The solids volume fraction in the recirculation region is almost constant, and increases with the mean solid volume fraction but decreases as the particle diameter increases (Fig. 10). A simple way to estimate the extension of the region in which the concentration field is affected by the step is purposed; such length is influenced by the expansion ratio and the particle diameter, while the effect of the mean solid volume fraction seems to be minor (Fig. 11b). Longer distance is required for achieving a fully-developed solids volume fraction profile rather that a constant pressure gradient (Fig. 9b and Fig. 11b).

Acknowledgement. This work has been supported by Regione Lombardia and CILEA Consortium through a LISA Initiative (Laboratory for Interdisciplinary Advanced Simulation) 2010 grant [link: http://lisa.cilea.it]. The authors would acknowledge Mike Malin of CHAM Ltd for useful discussions and comments.

\section{REFERENCES}

Badr, H.M., Habib, M.A., Ben-Mansour, R., Said, S.A.M., 2005. Numerical investigation of erosion threshold velocity in a pipe with sudden contraction. Computers and Fluids, 34, 721-742.

Badr, H.M., Habib, M.A., Ben-Mansour, R., Said, S.A.M., 2008. Erosion and penetration rates of a pipe protruded in a sudden contraction. Computers and Fluids, 37, 146-160.

Chen, L., Duan, Y., Pu, W., Zhao, C., 2009. CFD simulation of coal-water slurry flowing in horizontal pipelines. Korean J. Chem. Eng., 26, 1144-1154.

Clift, R., Grace, J.R., Weber, M.E., 1978. Bubbles, Drops and Particles. Academic Press, London.

Doron, P., Barnea, D., 1996. Flow pattern maps for solid-liquid flow in pipes. Int. J. Multiphase Flow, 22, 273-283.

Doron, P., Granica, D., Barnea, D., 1987. Slurry flow in horizontal pipes - experimental and modeling. Int. J. Multiphase Flow, 13, 535-547.

Duz, H., 2007. Theoretical Analysis of Sudden Expansion Fittings in Pneumatic Conveying System. M.Sc. Thesis, University of Gaziantep, Gaziantep, Turkey.
Enwald, H., Peirano, E., Almstedt, A.E., 1996. Eulerian twophase flow theory applied to fluidization. Int. J. Multiphase Flow, 22, 21-66.

Erdal, A., Anderssont, H.I., 1997. Numerical aspects of flow computation through orifices. Flow Meas. Instrum., 8, 27-37 .

Fessler, J.R., Eaton, K.E., 1997. Particle response in a planar sudden expansion flow. Exp. Thermal and Fluid Science, 15, 413-423.

Founti, M., Klipfel, A., 1998. Experimental and computational investigations of nearly dense two-phase sudden expansion flows. Exp. Thermal and Fluid Science, 17, 27-36.

Frawley, P., O’Mahony, A.P., Geron, M., 2010. Comparison of Lagrangian and Eulerian simulations of slurry flows in a sudden expansion. ASME J. Fluids Eng., 132, 9, 191-301.

Gillies, R.G., Shook, C.A., Xu, J., 2004. Modelling heterogeneous slurry flows at high velocities. Can. J. Chem. Eng., $82,1060-1065$.

Habib, M.A., Badr, H.M., Ben-Mansour, R., Said, S.A.M., 2004. Numerical calculations of erosion in an abrupt pipe contraction of different contraction ratios. Int. J. Num. Methods Fluids, 46, 19-35.

Habib, M.A., Badr, H.M., Ben-Mansour, R., Kabir, M.E., 2007. Erosion rate correlations of a pipe protruded in an abrupt pipe contraction. Int. J. Impact Eng., 34, 1350-1369.

Ishii, M., Mishima, K., 1984. Two-fluid model and hydrodynamic constitutive relations. Nuclear Engineering and Design, 82, 107-126.

Kaushal, D.R., Tomita, Y., 2003. Comparative study of pressure drop in multisized particulate slurry flow through pipe and rectangular duct. Int. J. Multiphase Flow, 29, 1473-1487 .

Kaushal, D.R., Sato, K., Toyota, T., Funatsu, K., Tomita, Y., 2005. Effect of particle size distribution on pressure drop and concentration profile in pipeline flow of highly concentrated slurry. Int. J. Multiphase Flow, 31, 809-823.

Koronaki, E.D., Liakos, H.H., Founti, M.A., Markatos, N.C., 2001. Numerical study of turbulent diesel flow in a pipe with sudden expansion. Appl. Math. Mod., 25, 319-333.

Lahiri, S.K., Ghanta, K.C., 2010. Slurry flow modeling by CFD. Chem. Ind. \& Chem. Eng. Quarterly, 16, 295-308.

Launder, B.E., Spalding, D.B., 1972. Mathematical Models of Turbulence. Academic Press, London.

Lin, C.X., Ebadian, M.A., 2008. A numerical study of developing slurry flow in the entrance region of a horizontal pipe. Computers and Fluids, 37, 965-974.

Ling, J., Skudarnov, P.V., Lin, C.X., Ebadian, M.A., 2003. Numerical investigations of solid-liquid slurry flows in a fully developed flow region. Int. J. Heat and Fluid Flow, 24, 389-398.

Marjoanovic, P., Levy, A., Mason, D.J., 1999. An investigation of the flow structure through abrupt enlargement of circular pipe. Powder Technology, 104, 296-303.

Matousek, V., 2000. Concentration distribution in pipeline flow of sand-water mixtures. J. Hydrol. Hydromech. 48, 180-196.

Messa, G.V., Malavasi, S., 2012. Solid-liquid slurry flow through an upward-facing step. In: Atti del XXXIII Convegno Nazionale di Idraulica e Costruzioni Idrauliche IDRA12 (CD-ROM), Università degli Studi di Brescia, Brescia, Italy, 10-15 September 2012.

Mohanarangam, K., Tu, T.J., 2009. Numerical study of particle turbulence interaction in liquid-particle flows. AIChE Journal, 55, 1298-1302. 
Pathak, M., 2011. Computational investigations of solid-liquid particle interaction in a two-phase flow around a ducted obstruction. J. Hydraulic. Res., 49, 96-104.

Poole, R.J., Escudier, M.P., 2004. Turbulent flow of viscoelastic liquids through an axisymmetric sudden expansion. J. Non-Newtonian Fluid Mech., 117, 25-46.

Roache, P.J., 1998. Verification and Validation in Computational Science and Engineering. Hermosa, Albuquerque.

Shaan, J., Sumner, R.J., Gillies, R.G., Shook, C.A., 2000. The effect of particle shape on pipeline friction for Newtonian slurries of fine particles. Can. J. Chem. Eng., 78, 717-725.

Shook, C.A., Bartosik, A.S., 1994. Particle-wall stresses in vertical slurry flows. Powder Technol., 81, 117-124.

Shook, C.A., Roco, M.C., 1991. Slurry Flow: Principles and Practice. Butterworth-Heinemann, Stoneham.

Siriboonluckul, N., Juntasaro, V., 2007. Turbulence modelling for wall-bounded particle-laden flow with separation. Int. Comm. Heat Mass Transfer, 34, 331-338.
Spalding, D.B., 1980. Numerical Computation of Multi-Phase Fluid Flow and Heat Transfer. In: Taylor, C., Morgan, K. (Eds.): Recent Advances in Numerical Methods in Fluids. Pineridge Press Limited, Swansea.

Vlasak, P., Chara, Z., 2011. Effect of particle size distribution and concentration on flow behavior of dense slurries. Particul. Sci. Technol, 29, 53-65.

Vlasak, P., Kysela, B., Chara, Z., 2012. Flow structure of coarse-grained slurry in a horizontal pipe. J. Hydrol. Hydromech., 60, 115-124.

Xiaowey, H., Liejin, G., 2010. Numerical investigation of catalyst-liquid slurry flow in the photocatalytic reactor for hydrogen production based on algebraic slip model. Int. J. Hydrogen Energy, 35, 7065-7072.

Received 7 March 2012 Accepted 23 January 2013

Note: Supplementary Figures (S1-S6) can be found in the web version of this article. 


\section{SUPPLEMENTARY MATERIAL}
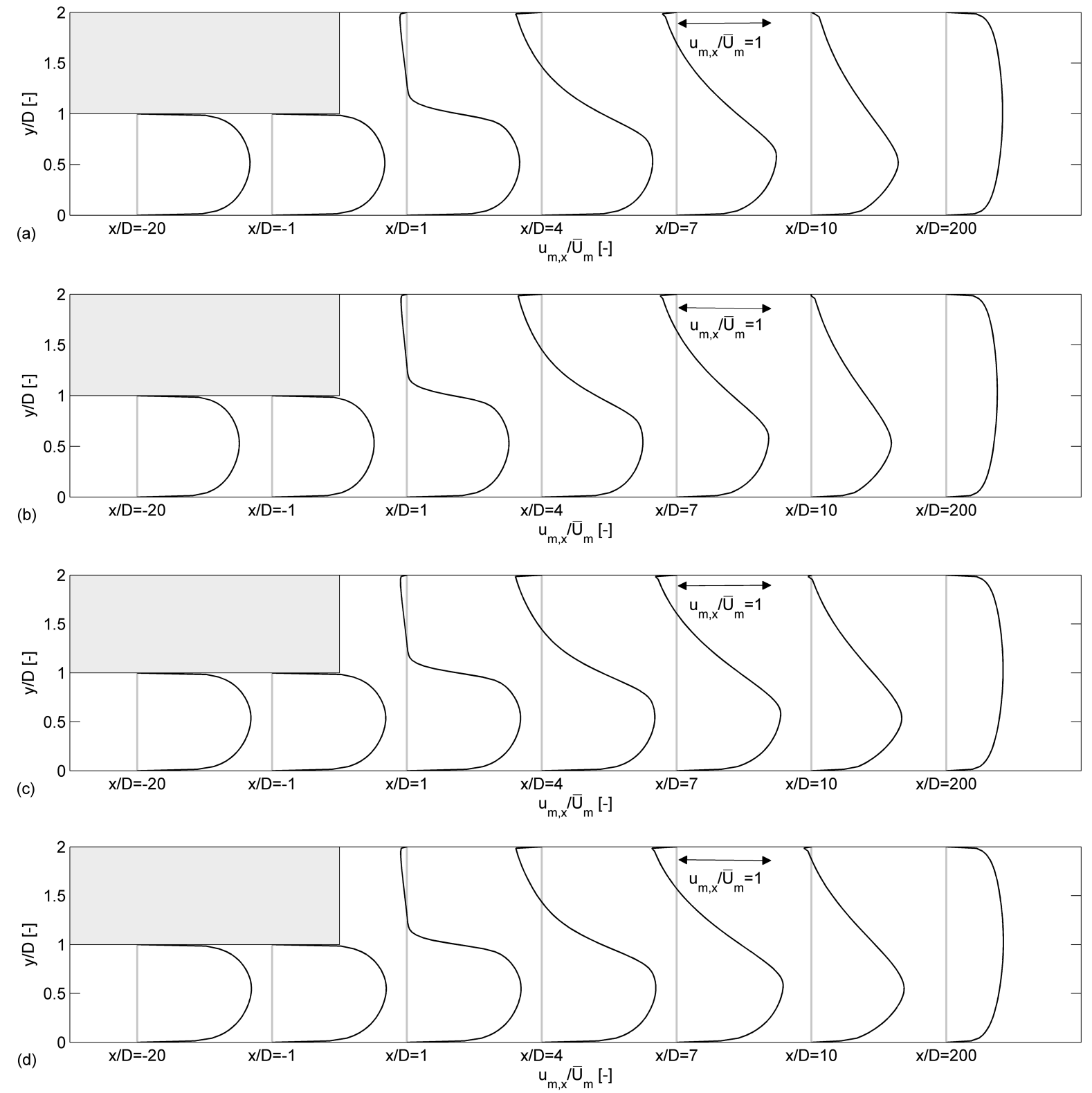

Fig. S1. Mixture velocity profiles for the case of $\beta=0.50$ and $d_{p}=125 \mu \mathrm{m}$ : (a) $\bar{\alpha}_{p}=0.05 ;$ (b) $\bar{\alpha}_{p}=0.10 ;$ (c) $\bar{\alpha}_{p}=0.15 ;$ (d) $\bar{\alpha}_{p}=0.20$. 
(a)

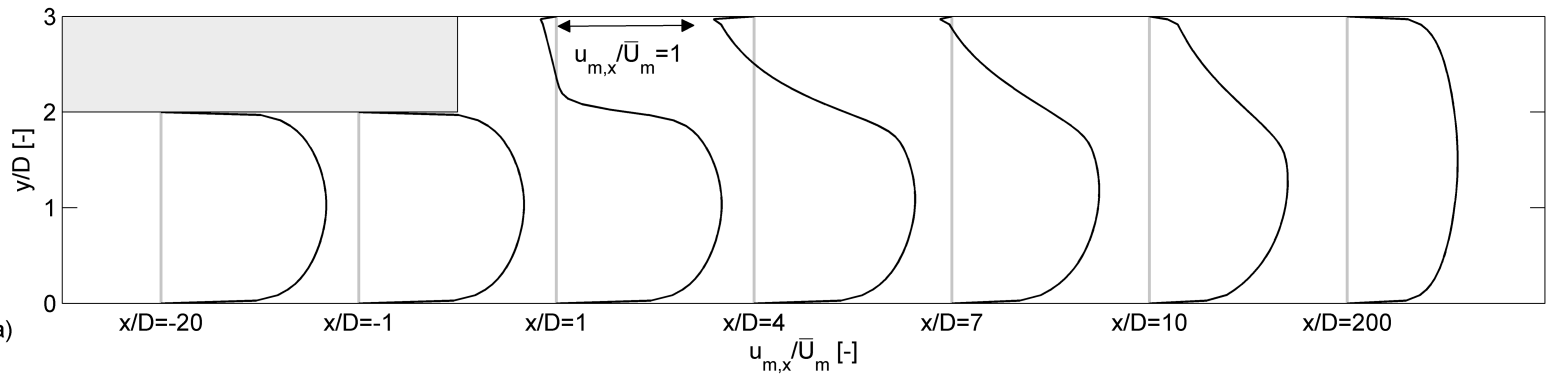

(b)

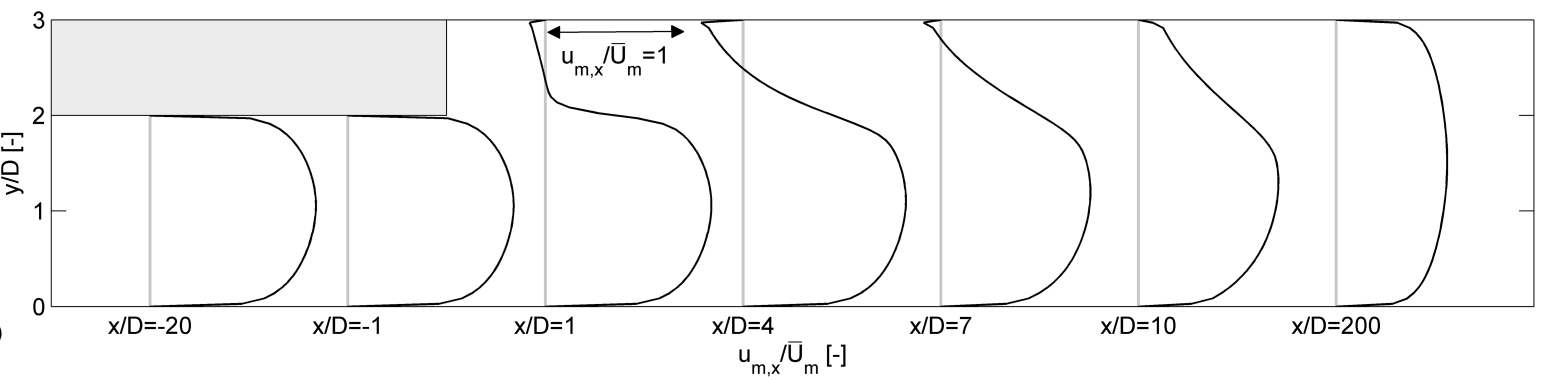

(c)

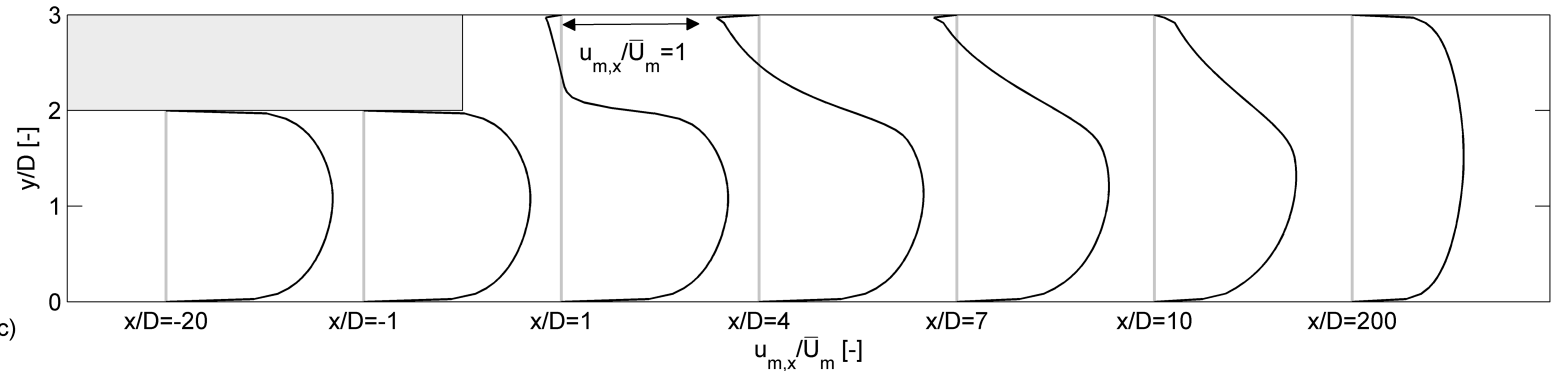

(d)

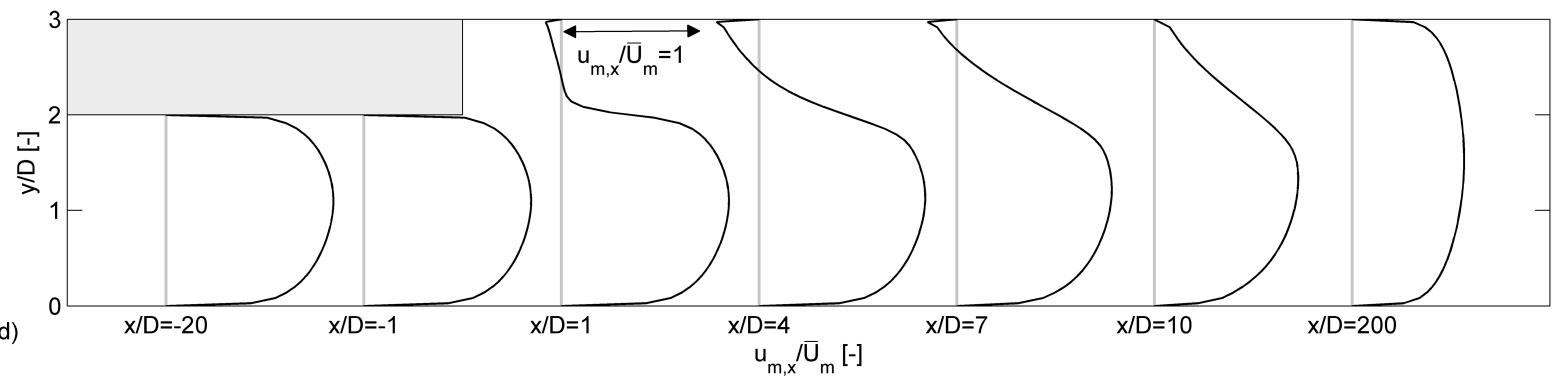

Fig. S2. Mixture velocity profiles for the case of $\beta=0.67$ and $d_{p}=125 \mu \mathrm{m}$ : (a) $\bar{\alpha}_{p}=0.05$; (b) $\bar{\alpha}_{p}=0.10 ;$ (c) $\bar{\alpha}_{p}=0.15 ;$ (d) $\bar{\alpha}_{p}=0.20$. 
(a)

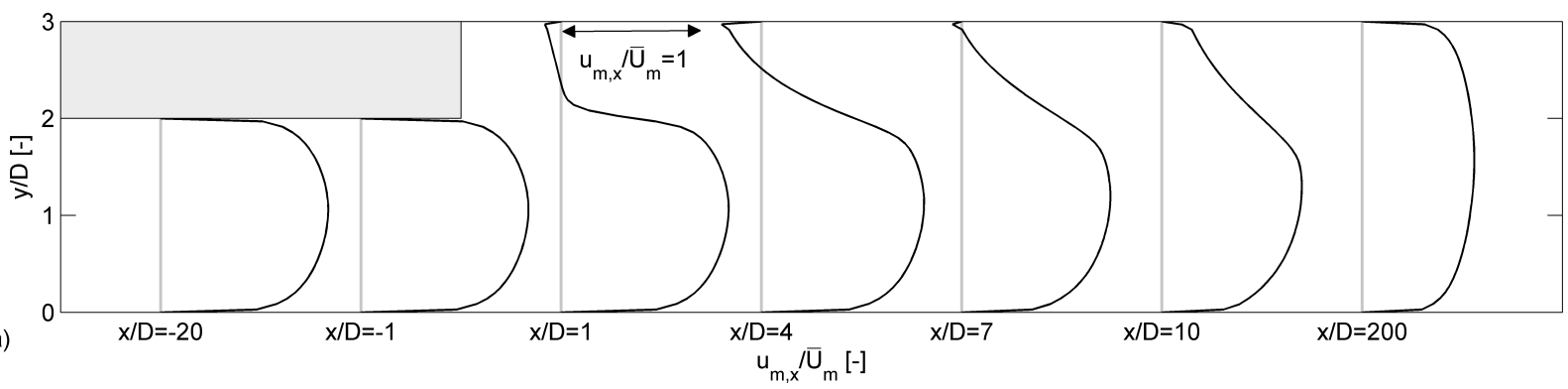

(b)

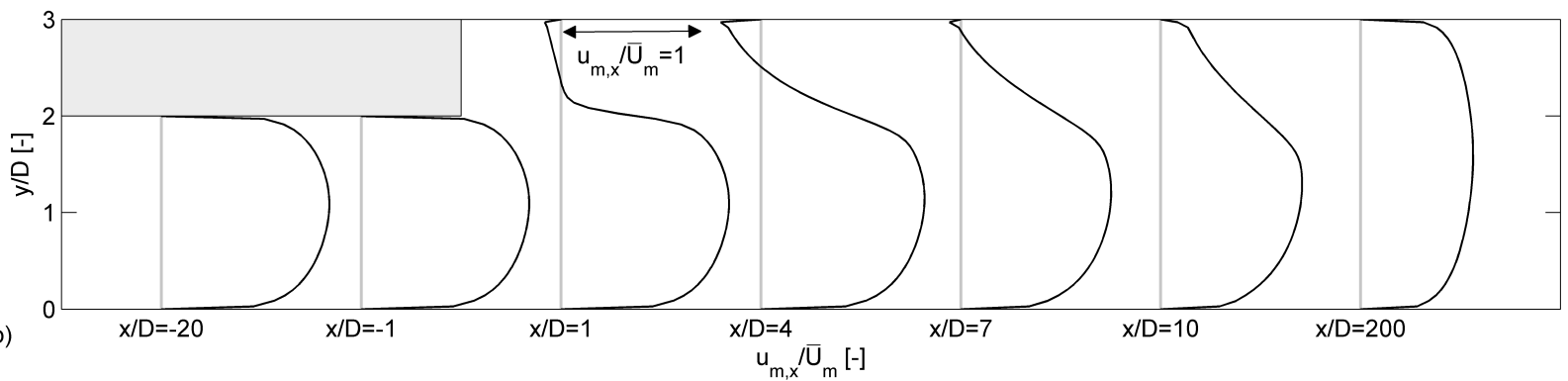

(c)

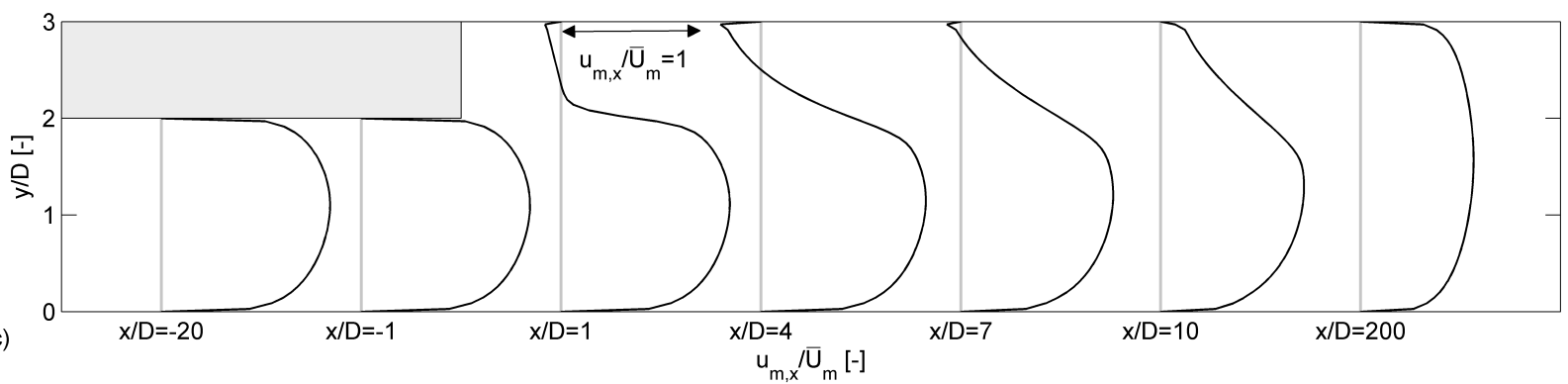

Fig. S3. Mixture velocity profiles for the case of $\beta=0.67$ and $d_{p}=440 \mu \mathrm{m}$ : (a) $\bar{\alpha}_{p}=0.05$; (b) $\bar{\alpha}_{p}=0.10 ;$ (c) $\bar{\alpha}_{p}=0.15$. 
(a)

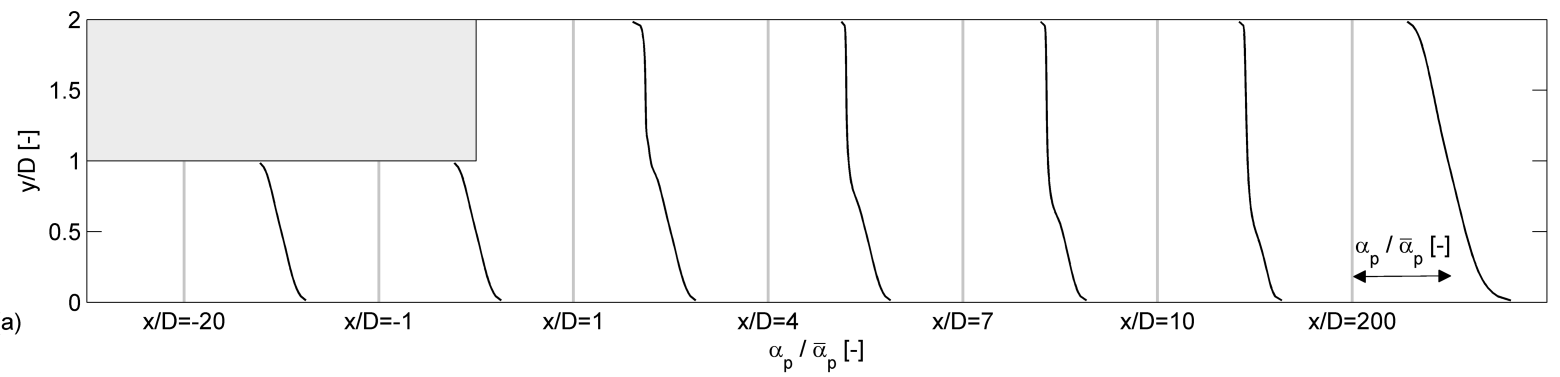

(b)

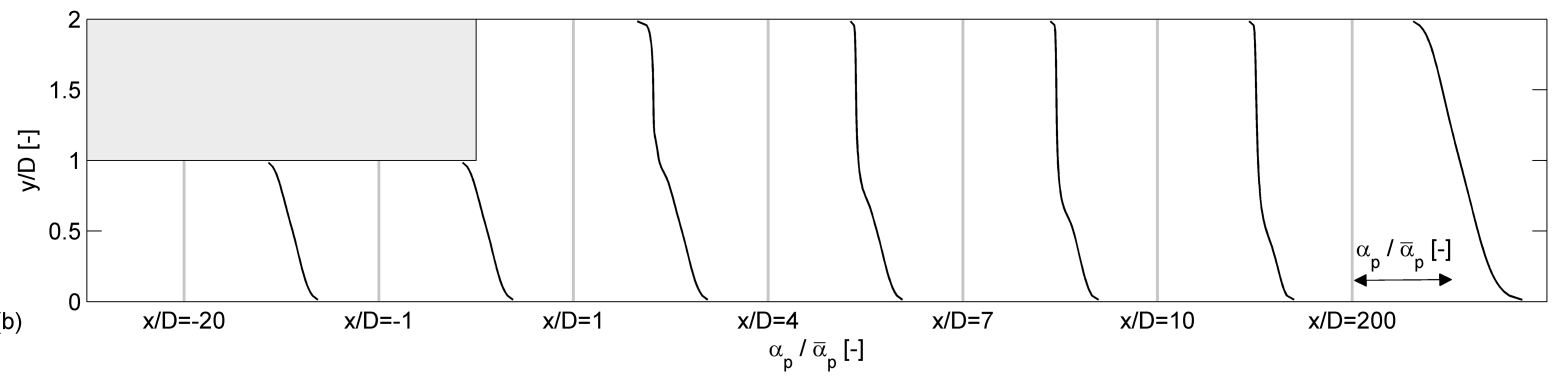

(c)
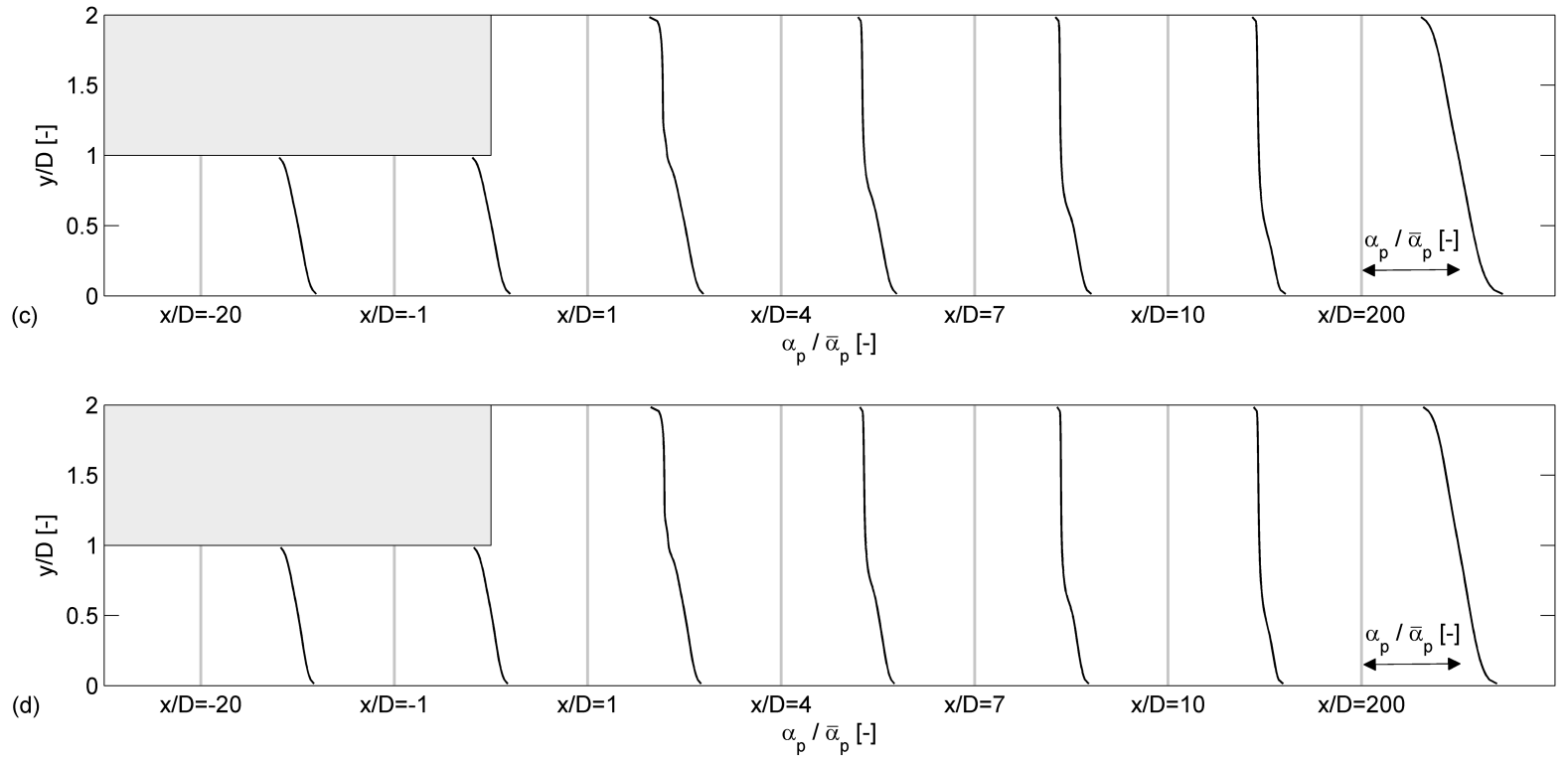

Fig. S4. Solids volume fraction profiles for the case of $\beta=0.50$ and $d_{p}=125 \mu \mathrm{m}:$ (a) $\bar{\alpha}_{p}=0.05 ;$ (b) $\bar{\alpha}_{p}=0.10 ;$ (c) $\bar{\alpha}_{p}=0.15 ;$ (d) $\bar{\alpha}_{p}=0.20$. 
(a)

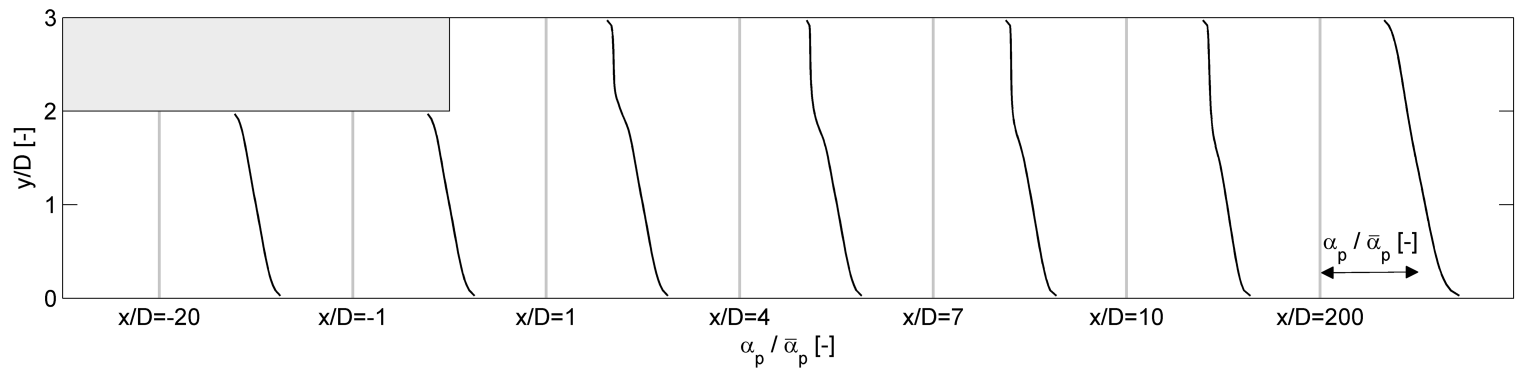

(b)

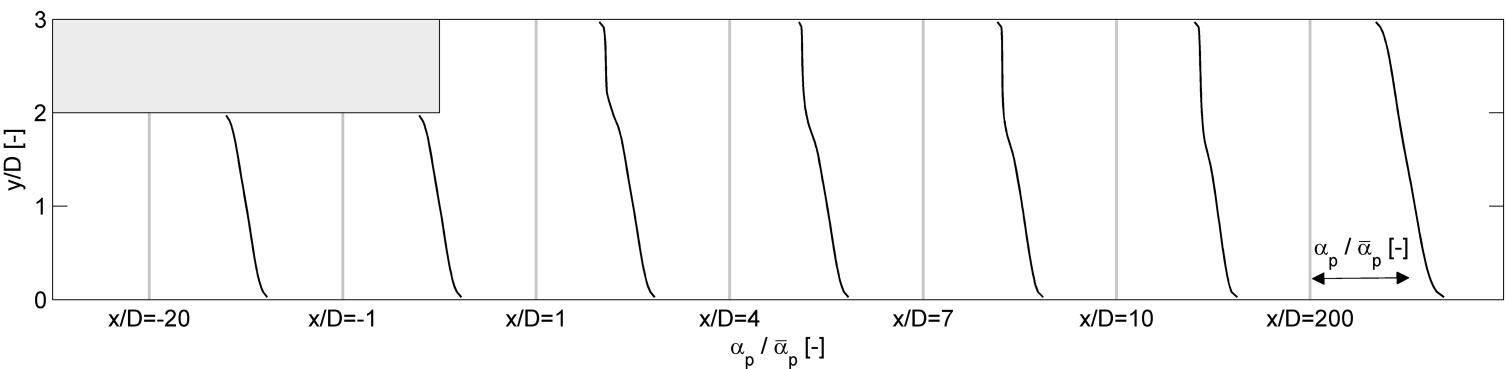

(c)

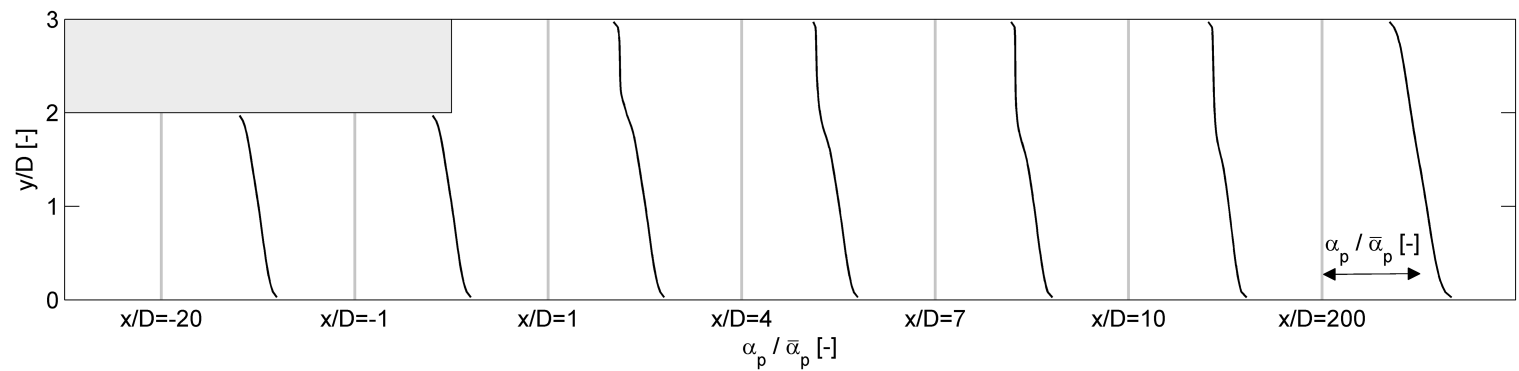

(d)

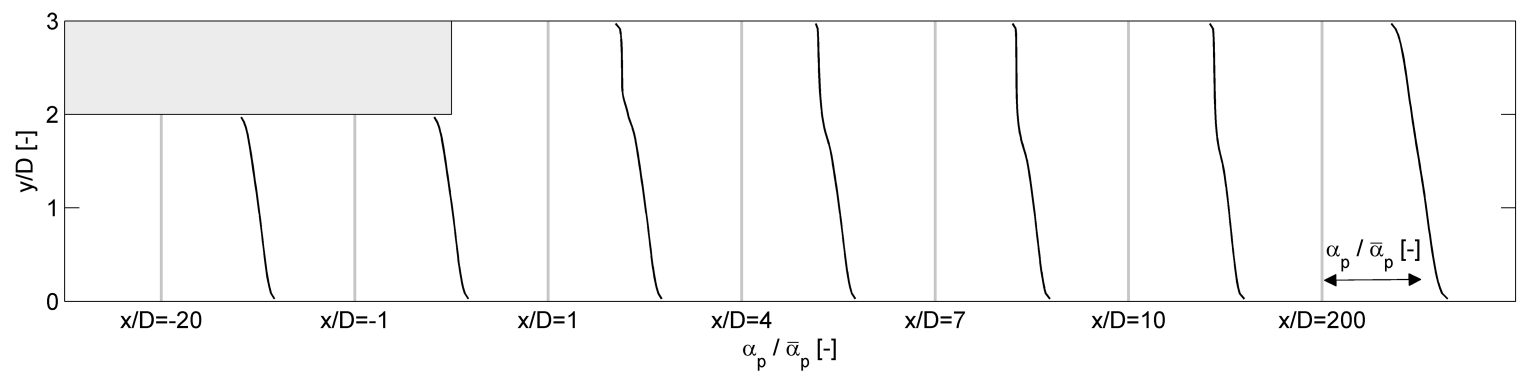

Fig. S5. Solids volume fraction profiles for the case of $\beta=0.67$ and $d_{p}=125 \mu \mathrm{m}:$ (a) $\bar{\alpha}_{p}=0.05 ;$ (b) $\bar{\alpha}_{p}=0.10 ;$ (c) $\bar{\alpha}_{p}=0.15 ;$ (d) $\bar{\alpha}_{p}=0.20$. 
(a)

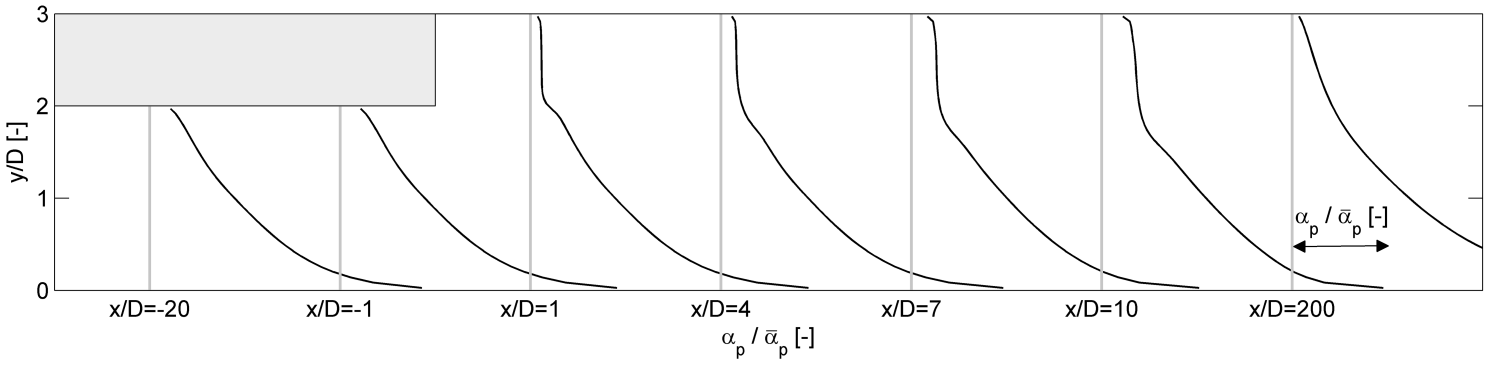

(b)

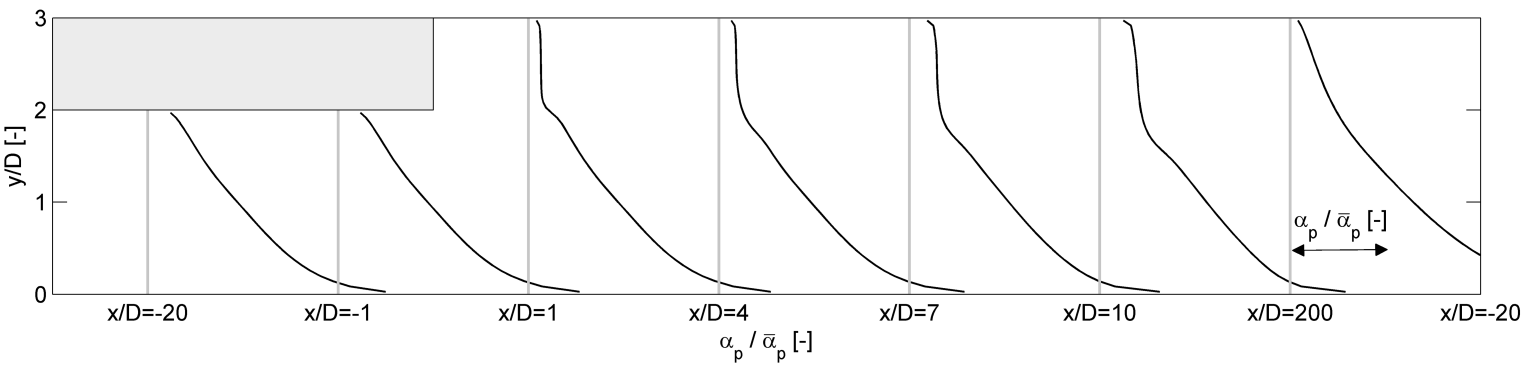

(a)

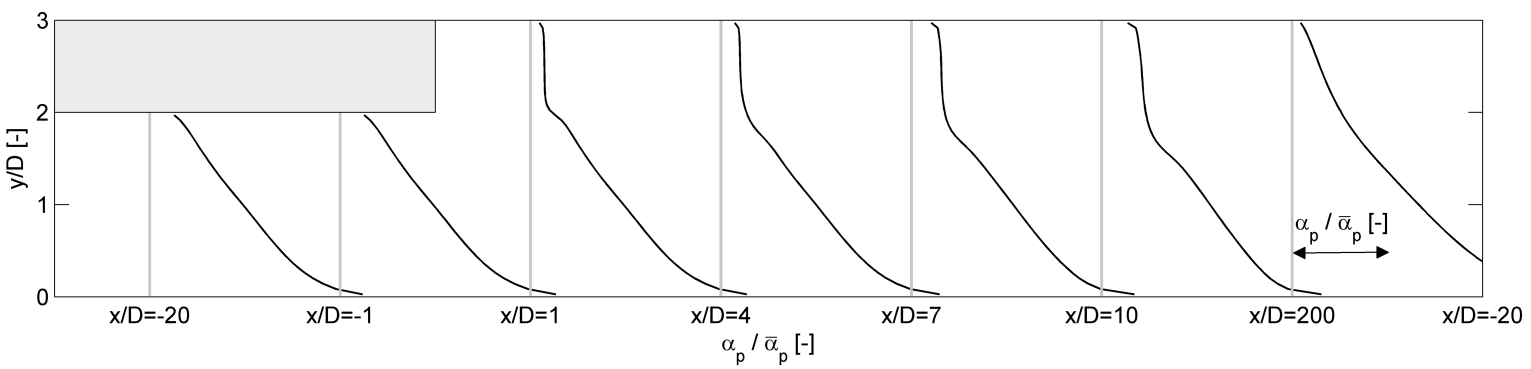

Fig. S6. Solids volume fraction profiles for the case of $\beta=0.67$ and $d_{p}=440 \mu \mathrm{m}$ : (a) $\bar{\alpha}_{p}=0.05 ;$ (b) $\bar{\alpha}_{p}=0.10 ;$ (c) $\bar{\alpha}_{p}=0.15$. 